\title{
Herschel-Bulkley Model of Blood Flow through a Stenosed Artery with the Effect of Chemical Reaction on Solute Dispersion
}

\author{
Siti Nurul Aifa Mohd Zainul Abidin, Nurul Aini Jaafar, Zuhaila Ismail* \\ Department of Mathematical Sciences, Faculty of Science, Universiti Teknologi \\ Malaysia, 81310 UTM Johor Bahru, Johor, Malaysia
}

Abstract A non-Newtonian mathematical model of blood flow described as the HershelBulkley fluid model in a stenosed artery is studied together with the effect of its chemical reaction. The expressions of the shear stress, velocity, mean velocity, and relative velocity in the plug and non-plug flow field were evaluated. The convective-diffusion equation was solved using the Taylor-Aris technique subjected to the relevant boundary condition in determining the concentration as well as the relative and effective axial diffusivity of the solute. The efficiency of the dispersion process was affected by the presence of chemical reactions and stenosis in blood flow. The normalised velocity decreased as the power-law index and yield stress increased. The height and length of the stenosis, as well as the power-law index, increased with an increase in the parameters of the chemical reaction rate. In contrast, the relative axial diffusivity and effective axial diffusivity showed a reverse behaviour. The existence of stenosis restricted the blood flow and drug dispersion. In short, this study improved the understanding of the physiological processes involved in the dispersion of drugs and nutrients in the circulatory system. Furthermore, it proved that the dispersion of a solute in the blood flow happened at a low shear rate through narrow arteries.

Keywords: Blood flow, Convective-diffusion equation, Chemical reaction, Herschel-Bulkley fluid, Stenosis.

*For correspondence: zuhaila@utm.my

Received: 26 Jan 2021

Accepted: 1 July 2021

(C) Copyright Abidin. This article is distributed under the terms of the Creative Commons Attribution License, which permits unrestricted use and redistribution provided that the original author and source are credited.

\section{Introduction}

The theory of dispersion has wide applications in different fields of science and engineering including chemical engineering [1], biomedical engineering [2], physiological fluid dynamics [3], and environmental sciences [4]. The concept of axial dispersion was first introduced by Taylor [5] who investigated theoretically and experimentally the solute dispersion in a straight tube moving at a mean velocity. $\mathrm{He}$ reported that the combination of molecular diffusion and the mean velocity eventually led the solute to diffuse at a molecular diffusivity of $D_{e f f}=a^{2} w_{m}{ }^{2} / 48 D_{m}$, where $D_{m}$ is the molecular diffusivity, $w_{m}$ is the mean axial velocity, and $a$ is the radius of the tube. Later, Aris [6] who executed the method of moments found that Taylor's dispersion theory was only valid when $D_{\text {eff }} \geq D_{m}$. Therefore, Aris enhanced the dispersion theory by including the effect of axial molecular diffusion, $D_{e f f}=D_{m}+a^{2} w_{m}{ }^{2} / 48 D_{m}$.

Various mathematical models have been proposed to study the dispersion of a solute in blood flow subjected to stenosis by using a cylindrical tube. The blood flow in a stenosed artery is distinct from 
normal arteries [7]. The abnormal growth of deposits such as fats, lipids, and cholesterol along the arterial wall can cause a reduction in the diameter of the artery, thus disturbing the normal flow of blood. The arterial wall becomes thickened and hardened with these accumulations. Consequently, a plaque is formed and leads to an artery narrowing that is generally referred to as atherosclerosis or stenosis. Sriyab [8] mentioned that the study of blood flow in a stenosed artery is vital for the understanding of circulatory diseases. According to Shah [9] as well as Mishra and Siddiqui [10], when blood flows through an artery with stenosis in certain regions, the issue of solute dispersion becomes more complicated. To date, numerous researchers have investigated and reported on the flow of blood through an artery with stenosis [11-15].

One of the essential characteristics during the dispersion phase is the impact of a chemical reaction on both the solute and blood in the arteries. The chemical reaction is known as protein binding. Protein binding can minimise blood flow diffusion by reducing the solute molecules. Kori [16] stated that the process of diffusion becomes more difficult when the fluid is chemically reactive. Many reactions take place in the human body, including phase exchange, partitioning, boundary uptake, and oxygen binding to haemoglobin [17]. These reaction processes may occur in the bulk flow or at the boundary of the wall. Bird et al. [18] observed that solute dispersion occurred simultaneously with a chemical reaction, such as ester hydrolysis and gas absorption in an agitated tank. Ratchagar and Kumar [19] delineated that the blood flow concentration reduced as the chemical reaction rate increased in a stenosed artery. Meanwhile, Mwapinga et al. [20] reaffirmed that chemical reactions reduced the mass concentration while the presence of stenosis affected the flow of blood.

In addition, Sharp [21] used Taylor-Aris's dispersion theory to study the shear-augmented dispersion in Casson, Bingham, and power-law fluids. He observed that an effective molecular diffusion relied on certain rheological parameters of the fluid, for instance, the yield stress for Bingham fluid and Casson fluid and the power-law index for power-law fluid. A fluid is said to be Newtonian if it can flow through a large artery (diameter $>3 \mathrm{~mm}$ ) at a high shear rate $\left(>100 \mathrm{~s}^{-1}\right)$. In contrast, it shows a non-Newtonian behaviour if it flows in a small arterial (diameter $<3 \mathrm{~mm}$ ) at low shear rates. As mentioned by Tu and Deville [22], the non-Newtonian Herschel-Bulkley $(\mathrm{H}-\mathrm{B})$ fluid is advantageous as it can be reduced to the Newtonian, power-law, and Bingham fluid by applying the appropriate parameters. Mazumdar [23] concurred with Tu and Deville [22] and stated that the non-Newtonian H-B fluid model would provide more specific results compared to the power-law fluid model due to the presence of yield stress, apparent fluid viscosity, and power-law index. Hence, due to the similarities, he concluded that the H-B fluid model is the best representative of the blood flow.

In another study, by considering blood as H-B fluid, Jaafar et al. [24] used Taylor-Aris's dispersion theory and studied the impact of chemical reactions on a uniform dispersion of a solute in blood flow. The axial diffusion of the solute in $\mathrm{H}-\mathrm{B}$ fluid was seen to be more significant compared to Casson fluids. Similarly, Mukahal et al. [25] analysed the advection and dispersion of a passive solute in the steady unidirectional flow of a thin uniform rivulet using Taylor-Aris's dispersion theory. In order to determine the timedependent relationship that enhanced the dispersion coefficient, Salerno et al. [26] used Taylor-Aris's dispersion theory with the Brinkman approach. Furthermore, Westerbeek et al. [27] also studied the reduction of Taylor-Aris dispersion by lateral mixing for the purpose of chromatographic applications. They demonstrated that the Taylor-Aris dispersion coefficient was reduced by a factor of 3 in a channel. Hence, based on previous works in the literature, there is a lack of studies on the steady dispersion of a solute in blood flow with the inclusion of a chemically active species in a stenosed artery using TaylorAris's dispersion theory. The study of solute dispersion in a non-Newtonian fluid is vital to produce realistic results that better represent physical problems. Furthermore, the knowledge of the rheological parameters is necessary to understand any effects of non-Newtonian rheology on solute dispersion. By solving this problem, the effective axial diffusivity of solute in narrow arteries can be predicted. Specifically, this study aimed to evaluate the effects of reactive chemical species in a stenosed artery using Taylor-Aris's theory that was only addressed individually in previous studies.

\section{Mathematical Formulation}

Consider a chemically reactive solute along a tube that is miscible in the fluid and undergoes a first-order 
reaction in the bulk flow. The blood flow is assumed to be steady, laminar, axially symmetrical and fully developed uni-directional.

\section{Governing Equations}

The cylindrical polar coordinates $(\bar{r}, \bar{\psi}, \bar{z})$ where $\bar{r}$ and $\bar{z}$ indicates the radial and axial coordinates and $\bar{\psi}$ is the azimuthal angle is considered. This works will ignore the fluid velocity in $\bar{r}$ direction as its magnitude is negligibly small and only accounts in a $\bar{z}$ direction. Hence, $\bar{u}_{\bar{r}}=\bar{u}_{\bar{\psi}}=0$ [28]. Due to the axial symmetry, the velocity $\bar{u}_{\bar{z}}$ is uniform and independent in both $\bar{\psi}$ and $\bar{z}$ directions. Thus, the continuity equation in the one-dimensional flow can be reduced as:

$$
\frac{\partial \bar{u}_{z}}{\partial \bar{z}}=0 .
$$

The motion is a steady flow which means there is no variation concerning time and the gravitational acceleration $\bar{g}_{\bar{r}}=\bar{g}_{\bar{\psi}}=\bar{g}_{\bar{z}}=0$ for the horizontal flow [29]. The simplified form in the axial and radial direction of momentum equations can be written as:

$$
\begin{gathered}
\frac{\partial \bar{p}}{\partial \bar{z}}=-\frac{1}{\bar{r}} \frac{\partial}{\partial \bar{r}}(\bar{r} \bar{\tau}), \\
\frac{\partial \bar{p}}{\partial \bar{r}}=0,
\end{gathered}
$$

where $\partial \bar{p} / \partial \bar{z}$ is the pressure gradient and $\partial / \partial \bar{r}$ is the changes in the radial coordinate. According to Singh et al. [30], the H-B fluid constitutive equation is indicated as:

$$
\frac{d \bar{u}}{d \bar{r}}=\left\{\begin{array}{cl}
-\frac{1}{\eta_{H}}\left(\bar{\tau}-\bar{\tau}_{y}\right)^{n}, & \text { if } \bar{\tau} \geq \bar{\tau}_{y} \text { and } \bar{r}_{p} \leq \bar{r} \leq \bar{R}(\bar{z}), \\
0 \quad, \text { if } \bar{\tau}<\bar{\tau}_{y} \text { and } 0 \leq \bar{r}<\bar{r}_{p},
\end{array}\right.
$$

where $\bar{\tau}$ is the shear stress, $\bar{\tau}_{y}$ is the yield stress, and $\eta_{H}$ is the H-B fluid viscosity coefficient with dimension $\left(M L^{-1} T^{-2}\right)^{n} T$ and $n$ is the power-law index. Equation (4) displays normal shear flow in the field when $\bar{\tau}>\bar{\tau}_{y}$ while plug flow field takes place when $\bar{\tau}<\bar{\tau}_{y}$. Bessonov et al. [31] stated that whenever the yield stress is greater than the shear stress, the fluid in the region will not flow but just merely be carried along by the fluid particles in the adjacent shear flow region. The equation of the transport of a species with first-order chemical reaction in the fluid is as follows:

$$
\begin{aligned}
& \frac{1}{\bar{r}} \frac{\partial}{\partial \bar{r}}\left(\bar{r} \frac{\partial \bar{C}_{1}}{\partial \bar{r}}\right)-\bar{R} \bar{C}_{1}=\frac{\hat{u}_{-}}{D_{m}} \frac{\partial \bar{C}_{1}}{\partial \tilde{z}}, \\
& \frac{1}{\bar{r}} \frac{\partial}{\partial \bar{r}}\left(\bar{r} \frac{\partial \bar{C}_{2}}{\partial \bar{r}}\right)-\bar{R} \bar{C}_{2}=\frac{\hat{u}_{+}}{D_{m}} \frac{\partial \bar{C}_{2}}{\partial \tilde{z}},
\end{aligned}
$$

where $\bar{C}_{1}$ is the solute concentration at plug flow field, $\bar{C}_{2}$ is the solute concentration at the outer flow field, $\bar{u}_{-}$is the plug flow velocity, $\bar{u}_{+}$is the outer flow velocity, $\bar{u}_{m}$ is the mean velocity, $\bar{R}$ is the parameter of the chemical reaction rate, $\breve{u}_{-}=\bar{u}_{-}-\bar{u}_{m}$ is the relative velocity in the plug flow field and $\breve{u}_{+}=\bar{u}_{+}-$ $\bar{u}_{m}$ is the relative velocity in the non-plug flow field.

\section{Initial and Boundary Conditions}

Equations (2) define the nonlinear system of a differential equation for the unknown shear stress $\bar{\tau}$ subject to the following boundary condition: 
$\bar{\tau}$ is finite at $\bar{r}=0$.

For the unknown velocity $\bar{u}$, there is a no-slip condition at the wall of the circular pipe. As the fluid is viscous, it sticks to the pipe wall and thus the no-slip boundary condition is given by:

$$
\bar{u}=0 \text { at } \bar{r}=\bar{R}(\bar{z})
$$

The boundary conditions in the plug and non-plug flow fields for solute concentration are as follows:

$$
\begin{aligned}
& \frac{\partial \bar{C}_{1}}{\partial \bar{r}}=0 \text { at } \bar{r}=0, \\
& \bar{C}_{1}=0 \text { at } \bar{r}=0 . \\
& \frac{\partial \bar{C}_{2}}{\partial \bar{r}}=0 \text { at } \bar{r}=\bar{R}(\bar{z}), \\
& \bar{C}_{2}=\bar{C}_{p} \text { at } \bar{r}=\bar{r}_{p} .
\end{aligned}
$$

where $\bar{C}_{p}$ is the solute concentration at the plug flow field.

\section{Geometry of Stenosis}

Following Chitra and Karthikeyan [32], the geometry of the stenosis in the dimensional form may be written as:

$$
\bar{R}(\bar{z})=\left\{\begin{array}{cl}
R_{0}-\bar{\delta}\left[1+\cos \left(\frac{2 \pi}{l_{0}}\left(\bar{z}-d-\frac{l_{0}}{2}\right)\right)\right], & d \leq \bar{z} \leq d+l_{0}, \\
R_{0}, & \text { Otherwise }
\end{array}\right.
$$

where $R_{0}$ is the arterial radius, $\bar{\delta}$ is the height of stenosis, $\bar{l}_{0}$ is the length of a stenosis, $\bar{z}$ is the longitudinal (axial) distance, $\bar{r}$ is the transverse (radial) distance, $\bar{d}$ is the stenosis location, $\bar{L}$ is the artery length and $\bar{R}(\bar{z})$ is the stenotic artery radius.

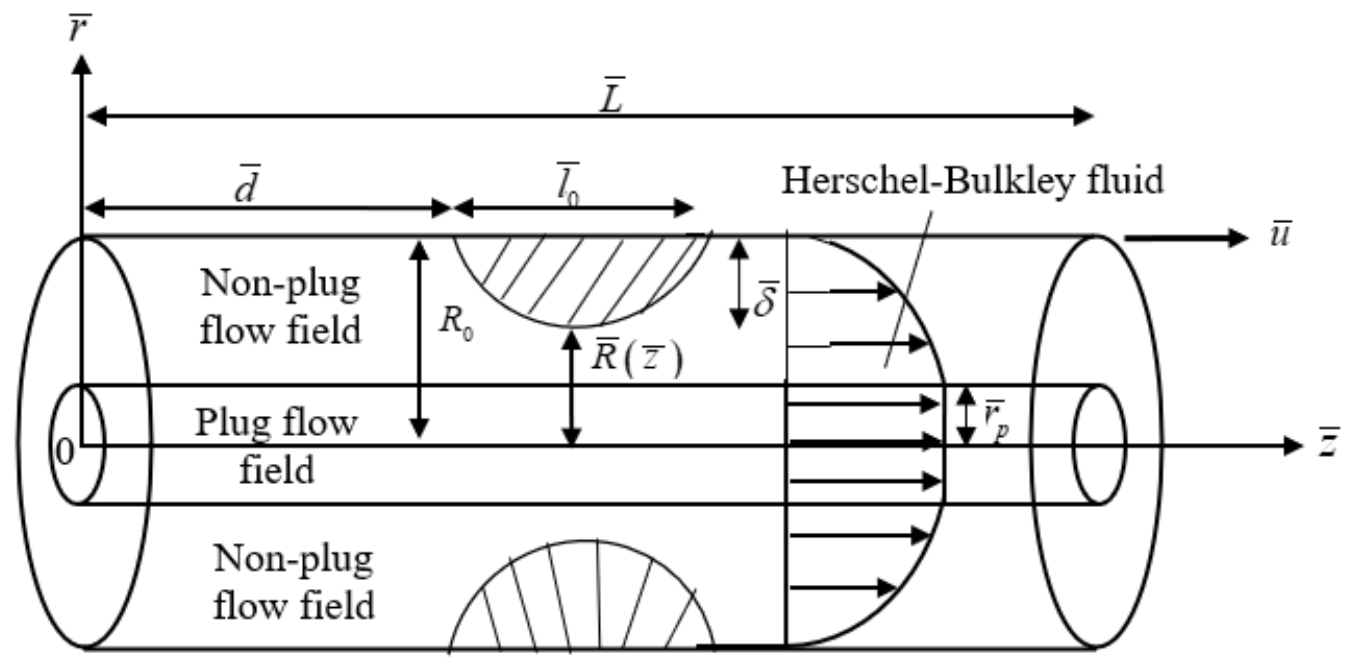

Figure 1. The geometry of the stenosis in an arterial segment. 
The introduction of some of the non-dimensional variables:

$$
\begin{gathered}
C=\frac{\bar{C}}{\bar{C}_{0}}, u=\frac{\bar{u}}{\bar{u}_{0}}, u_{+}=\frac{\bar{u}_{+}}{\bar{u}_{0}}, u_{-}=\frac{\bar{u}_{-}}{\bar{u}_{0}}, u_{m}=\frac{\bar{u}_{m}}{\bar{u}_{0}}, r=\frac{\bar{r}}{R_{0}}, r_{p}=\frac{\bar{r}_{p}}{R_{0}}, z=\frac{D_{m} \tilde{z}}{R_{0}^{2} \bar{u}_{0}}, \tau=\frac{\bar{\tau}}{\left(\eta \bar{u}_{0} / R_{0}\right)^{\frac{1}{n}}}, \\
\tau_{y}=\frac{\bar{\tau}_{y}}{\left(\eta \bar{u}_{0} / R_{0}\right)^{\frac{1}{n}}}, R(z)=\frac{\bar{R}(\bar{z})}{R_{0}}, R=\sqrt{\frac{R_{0}^{2} \bar{R}}{D_{m}}}, P e=\frac{\bar{R}(\bar{z}) \bar{u}_{m}}{D_{m}}, \delta=\frac{\bar{\delta}}{R_{0}}, l_{0}=\frac{\bar{l}_{0}}{R_{0}}, z=\frac{\bar{z}}{R_{0}},
\end{gathered}
$$

where $\bar{u}_{0}$ is the fluid characteristics velocity (centreline velocity), $u$ is the velocity, $u_{+}, u_{-}$is the non-plug and plug flow velocities, $u_{m}$ is the average velocity, $r$ is the transverse (radial) distance, $r_{p}$ is the radius of the plug core field, $z$ is the longitudinal (axial) distance, and $P e$ is the modified Péclet number. Using non-dimensional variables in (14), the corresponding equations and boundary conditions from Equations (1) until (13) are as follows,

$$
\begin{gathered}
\frac{\partial u_{z}}{\partial z}=0, \\
\frac{\partial p}{\partial z}=-\frac{1}{r} \frac{\partial}{\partial r}(r \tau), \\
\frac{\partial p}{\partial r}=0, \\
R(z)=\left\{\begin{array}{c}
-\left(\tau-\tau_{y}\right), \text { if } \tau \geq \tau_{y} \text { and } r_{p} \leq r \leq R(z), \\
0, \text { if } \tau<\tau_{y} \text { and } 0 \leq r<r_{p}, \\
\frac{1}{r} \frac{\partial}{\partial r}\left(r \frac{\partial C_{1}}{\partial r}\right)-R C_{1}=\frac{\hat{u}_{-}}{D_{m}} \frac{\partial C_{1}}{\partial z}, \\
\frac{1}{r} \frac{\partial}{\partial r}\left(r \frac{\partial C_{2}}{\partial r}\right)-R C_{2}=\frac{\hat{u}_{+}}{D_{m}} \frac{\partial C_{2}}{\partial z}, \\
\tau \text { is finite at } r=0, \\
u=0 \text { at } r=R(z), \\
\frac{\partial C_{1}}{\partial r}=0 \text { at } r=0, \\
C_{1}=0 \text { at } r=0, \\
\frac{\partial C_{2}}{\partial r}=0 \text { at } r=R(z), \\
C_{2}=C_{p} \text { at } r=r_{p}, \\
\left.1+\cos \left(\frac{2 \pi}{l_{0}}\left(z-d-\frac{l_{0}}{2}\right)\right)\right], \quad d \leq z \leq d+l_{0},
\end{array} \quad\right. \text { Otherwise. }
\end{gathered}
$$

\section{Method of Solution}

To solve equation (13) subject to the boundary condition equation (8), the shear stress $\bar{\tau}$ should be obtained by integrating equation (2) with respect to $\bar{r}$ subject to the boundary condition equation (7) Hence, the expression for the shear stress is obtained as follows: 


$$
\bar{\tau}=-\frac{\bar{r}}{2} \frac{d \bar{p}}{d \bar{z}}
$$

Since $\left(\bar{\tau}_{y} / \bar{\tau}\right) \ll 1$, disregard the terms $\left(\bar{\tau}_{y} / \bar{\tau}\right)^{3}$ and higher powers of $\left(\bar{\tau}_{y} / \bar{\tau}\right)$, the constitutive equation in Equation (4) can be summarised as follows:

$$
\frac{d \bar{u}}{d \bar{r}}=-\frac{1}{\eta_{H}}\left(-\frac{1}{2} \frac{d \bar{p}}{d \bar{z}}\right)^{n}\left(\bar{r}^{n}-n \bar{r}_{p} \bar{r}^{n-1}+\frac{n(n-1)}{2} \bar{r}_{p}^{2} \bar{r}^{n-2}\right), \quad \text { if } \bar{\tau} \geq \bar{\tau}_{y} \text { and } \bar{r}_{p} \leq \bar{r} \leq \bar{R}(\bar{z}) .
$$

In order to differentiate between the velocity in the non-plug flow field and plug flow field, the velocity in the non-plug flow field is defined as $\bar{u}_{+}(\bar{r})$ and is given by:

$$
\begin{array}{r}
\bar{u}_{+}(\bar{r})=\frac{1}{(n+1) \bar{\eta}_{H}}\left(-\frac{1}{2} \frac{d \bar{p}}{d \bar{z}}\right)^{n}\left[\bar{R}(\bar{z})^{n+1}-\bar{r}^{n+1}-(n+1) \bar{r}_{p}\left(\bar{R}(\bar{z})^{n}-\bar{r}^{n}\right)+\frac{n(n+1)}{2} \bar{r}_{p}^{2}\left(\bar{R}(\bar{z})^{n-1}-\bar{r}^{n-1}\right)\right], \\
\text { if } \bar{\tau} \geq \bar{\tau}_{y} \text { and } \bar{r}_{p} \leq \bar{r} \leq \bar{R}(\bar{z}),
\end{array}
$$

where $\bar{r}_{p}$ is equal to the yield stress over a pressure gradient that can be described as the plug core radius:

$$
\bar{r}_{p}=-\frac{2 \bar{\tau}_{y}}{d \bar{p} / d \bar{z}}
$$

By evaluating Equation (30) at $\bar{r}=\bar{r}_{p}$, the plug flow field velocity can be described as:

$$
\begin{array}{r}
\bar{u}_{-}\left(\bar{r}_{p}\right)=\frac{1}{(n+1) \bar{\eta}_{H}}\left(-\frac{1}{2} \frac{d \bar{p}}{d \bar{z}}\right)^{n}\left[\bar{R}(\bar{z})^{n+1}-(n+1) \bar{r}_{p} \bar{R}(\bar{z})^{n}+\frac{n(n+1)}{2} \bar{r}_{p}^{2} \bar{R}(\bar{z})^{n-1}-\frac{n(n-1)}{2} \bar{r}_{p}^{n+1}\right], \\
\text { if } \bar{\tau}<\bar{\tau}_{y} \text { and } 0 \leq \bar{r}<\bar{r}_{p} .
\end{array}
$$

The dimensional form of the mean velocity, $\bar{u}_{m}$ in a circular pipe, is:

$$
\bar{u}_{m}=\bar{u}_{H} A\left(\bar{r}_{p}\right)
$$

where

$$
\bar{u}_{H}=\frac{\bar{R}(\bar{z})^{n+1}}{(n+3) \eta_{H}}\left(-\frac{1}{2} \frac{d \bar{p}}{d \bar{z}}\right)^{n}
$$

and

$$
A\left(\bar{r}_{p}\right)=1-\frac{n(n+3)}{(n+2)} \frac{\bar{r}_{p}}{\bar{R}(\bar{z})}+\frac{n(n+3)(n-1)}{2(n+1)} \frac{\bar{r}_{p}^{2}}{\bar{R}(\bar{z})^{2}}-\frac{\left(n^{4}+2 n^{3}-5 n^{2}-6 n+4\right)}{2(n+1)(n+2)} \frac{\bar{r}_{p}^{n+3}}{\bar{R}(\bar{z})^{n+3}}
$$

When $\bar{r}_{p}=0$ and $n=1$, Equation (33) is deduced as the Newtonian fluid mean velocity. In order to compute the values of blood velocity, the normalised velocity is defined to reduce some physical quantities for simpler evaluation. Using a similar approach by Sharp [16] as shown in Figure 3, the H-B normalised velocity profile can be derived by dividing the velocity $\bar{u}$, $\left(\bar{u}=\bar{u}_{-}\left(\bar{r}_{p}\right)\right.$ if $0 \leq \bar{r} \leq \bar{r}_{p}$ and $\bar{u}=$ $\bar{u}_{+}(\bar{r})$ if $\bar{r}_{p} \leq \bar{r} \leq \bar{R}(\bar{z})$ in both outer and plug flow fields with mean velocity, $\bar{u}_{m}$. It is defined as:

$$
\text { The normalised velocity of blood }=\frac{\bar{u}}{\bar{u}_{m}} \text {. }
$$

The non-dimensional velocity of blood in the outer flow field is given by: 


$$
\begin{gathered}
u_{+}(r)=1-\frac{r^{n+1}}{R(z)^{n+1}}-(n+1) \frac{r_{p}}{R(z)}\left(1-\frac{r^{n}}{R(z)^{n}}\right)+\frac{n(n+1)}{2} \frac{r_{p}^{2}}{R(z)^{2}}\left(1-\frac{r^{n-1}}{R(z)^{n-1}}\right), \\
\text { if } \tau \geq \tau_{y} \text { and } r_{p} \leq r \leq R(z),
\end{gathered}
$$

and the non-dimensional velocity of blood in the plug flow field is:

$$
u_{-}\left(r_{p}\right)=1-(n+1) \frac{r_{p}}{R(z)}+\frac{n(n+1)}{2} \frac{r_{p}^{2}}{R(z)^{2}}-\frac{n(n-1)}{2} \frac{r_{p}^{n+1}}{R(z)^{n+1}}, \quad \text { if } \tau<\tau_{y} \text { and } 0 \leq r<r_{p} .
$$

The mean velocity in non-dimensional is given as:

$$
\begin{array}{r}
u_{m}=\frac{(n+1)}{(n+3)}\left(1-\frac{n(n+3)}{(n+2)} \frac{r_{p}}{R(z)}-\frac{n(n+3)}{(n+2)} \frac{r_{p}}{R(z)}+\frac{n(n-1)(n+3)}{2(n+1)} \frac{r_{p}{ }^{2}}{R(z)^{2}}\right. \\
\left.-\frac{\left(n^{4}+2 n^{3}-5 n^{2}-6 n+4\right)}{2(n+1)(n+2)} \frac{r_{p}{ }^{n+3}}{R(z)^{n+3}}\right) .
\end{array}
$$

The plug flow field of relative velocity in the non-dimensional form is:

$$
\hat{u}_{-}=\frac{2}{n+3}-\frac{2(n+1)}{n+2} \frac{r_{p}}{R(z)}+\frac{n r_{p}{ }^{2}}{R(z)^{2}}-\frac{n(n-1)}{2} \frac{r_{p}^{n+1}}{R(z)^{n+1}}+\frac{\left(n^{4}+2 n^{3}-5 n^{2}-6 n+4\right)}{2(n+2)(n+3)} \frac{r_{p}^{n+3}}{R(z)^{n+3}},
$$

and the non-plug flow field of relative velocity is:

$$
\begin{array}{r}
\hat{u}_{-}=\frac{2}{n+3}+\frac{n r_{p}{ }^{2}}{R(z)^{2}}-\frac{2(n+1)}{n+2} \frac{r_{p}}{R(z)}+\frac{\left(n^{4}+2 n^{3}-5 n^{2}-6 n+4\right)}{2(n+2)(n+3)} \frac{r_{p}^{n+3}}{R(z)^{n+3}}-\frac{r^{n+1}}{R(z)^{n+1}}+(n+1) \frac{r^{n} r_{p}}{R(z)^{n+1}} \\
-\frac{n(n-1)}{2} \frac{r^{n-1} r_{p}{ }^{2}}{R(z)^{n+1}}
\end{array}
$$

Equation (19) is solved analytically pertaining to the boundary conditions in (23) and (24) to get the solute concentration in the plug flow field in terms of modified Bessel function:

$$
C_{1}=\frac{2 u_{H}}{(n+1) R \sqrt{D_{m}}}\left(\frac{\partial C_{1}}{\partial z}\right)\left\{J_{0}\left(R^{1 / 2} r_{p}\right)-1\right\} \hat{u}_{-}
$$

where $J_{0}$ is the first kind of zeroth-order modified Bessel function.

Due to the complexity to solve Equation (20) analytically, it is solved numerically using the Mathematica software with the boundary conditions (25) and (26). The expression of solute concentration in the nonplug flow field is very lengthy, thus it is not presented here. The flux (flow rate) of solute over a crosssection of the geometry in a circular pipe flow at constant, $z$ is:

$$
q=\frac{1}{\pi(R(z))^{2}}\left\{\int_{0}^{r_{p}}\left(\hat{u}_{-} C_{1}-D_{m} \frac{\partial C_{1}}{\partial z}\right) 2 \pi r d r+\int_{r_{p}}^{R(z)}\left(\hat{u}_{+} C_{2}-D_{m} \frac{\partial C_{2}}{\partial z}\right) 2 \pi r d r\right\}
$$

Hence, the flux of solute can be simplified as:

$$
q=-D_{m} \frac{\partial C}{\partial z}\left(1+\frac{P e^{2}}{48} \frac{E\left(r_{p}\right)}{A^{2}\left(r_{p}\right)}\left(\frac{1}{R(z)^{4}}\right)\right)
$$


where

$$
P e=\frac{R(z) \bar{u}_{H} A\left(r_{p}\right)}{D_{m}}=\frac{R(z) \bar{u}_{m}}{D_{m}}
$$

and

$$
E\left(r_{p}\right)=\left\{\int_{0}^{r_{p}} \hat{u}_{-} C_{1} r d r+\int_{r_{p}}^{R(z)} \hat{u}_{+} C_{2} r d r\right\} .
$$

The solute effective axial diffusivity can be obtained by dividing Equation (44) with $-(\partial C / \partial z)$ as follows:

$$
D_{e f f}=-\frac{q}{\partial C / \partial z}=D_{m}\left[1+\frac{P e^{2}}{48} \frac{E\left(r_{p}\right)}{A^{2}\left(r_{p}\right)}\left(\frac{1}{R(z)^{4}}\right)\right]
$$

It can be re-written as:

$$
\frac{D_{e f f}}{D_{m}}=\left[1+\frac{P e^{2}}{48} \frac{E\left(r_{p}\right)}{A^{2}\left(r_{p}\right)}\left(\frac{1}{R(z)^{4}}\right)\right]
$$

\section{Results and discussion}

The objective of the study was to analyse the behaviour of the blood flow for steady dispersion through a narrow circular pipe to simulate the presence of stenosis and reactive species in the bulk flow. The study also aimed to portray the non-Newtonian behaviour and the effects of the various physical parameters on the velocity distribution of blood as well as the relative and effective axial diffusivity of the solute. The normalised velocity of blood was calculated in dimensional terms where the relative axial diffusivity and effective axial diffusivity of the solute was evaluated in non-dimensional terms for simplicity. The range of the parameter values used in this study was as follows: yield stress $r_{p}: 0-0.2$ [33]; power-law index $n: 0.6-1.4$ [34]; rate of chemical reaction parameter $R: 0-10$ [35;36]; stenosis height $\delta: 0.05-0.25$ [19]; longitudinal (axial) distance $z: 3.9-5$ [37], and Péclet number Pe : $4-20$ [38]. The analysis results in Figure 2-14 and Table 1-2 were computed and generated using Mathematica software.

\section{Normalised Velocity Distribution}

Figure 2 depicts the normalised velocity profiles of different fluids when $\bar{l}_{0}=3, \bar{d}=2, \bar{z}=4$, and $\bar{\delta}=$ 0.01 . It shows the effect of plug core radius and power-law index on the normalised velocity of the blood flow in the stenosed artery. The physically meaningless negative values of the parameter $\bar{r}$ are used to show the symmetry of the normalised velocity profile at the centreline, $\bar{r}=0$. The normalised velocity decreases when the power-law index $n$ and yield stress increase. When $n=0.95$ for (H-B fluid), the normalised velocity is higher than the normalised velocity when $n=1$ (Bingham fluid). The normalised velocity when $\bar{r}_{p}=0.04$ (H-B fluid) is lower than the normalised velocity when $\bar{r}_{p}=0$ (power-law fluid). An increase in the yield stress can lead to a higher accumulation of red blood cells at the centre of the artery, hence reducing the velocity of blood flow. It can be seen that the normalised velocity of the Newtonian fluid model is marginally higher than $\mathrm{H}-\mathrm{B}$ and Bingham fluid models and slightly higher than the power-law fluid model. However, the Newtonian and power-law fluids are suitable for larger arteries without yield stress $\left(\bar{r}_{p}=0\right)$ when the red blood cells do not accumulate in the central area, while $\mathrm{H}-\mathrm{B}$ and Bingham fluids are good for narrow arteries.

According to Taylor [5], the dispersion process can be affected by the normalised velocity profile. A higher normalised velocity profile achieve a more effective diffusivity of solute. $\mathrm{H}-\mathrm{B}$ fluid model consists 
of the power-law index $n$ that can decrease the viscosity and increase the velocity. It is clear that the normalised velocity profile of $\mathrm{H}-\mathrm{B}$ fluid is higher than Bingham fluid when $n=0.95$. Thus, it can be concluded that $\mathrm{H}-\mathrm{B}$ fluid is a better fluid model to be considered in a narrow artery at a low shear rate as it is more closely related to the actual physiology and it can help the medicine to move faster in the blood flow. Interestingly, the normalised velocity profile is in good agreement with Figure 2 of Sankar et al. [39] in the absence of stenosis.

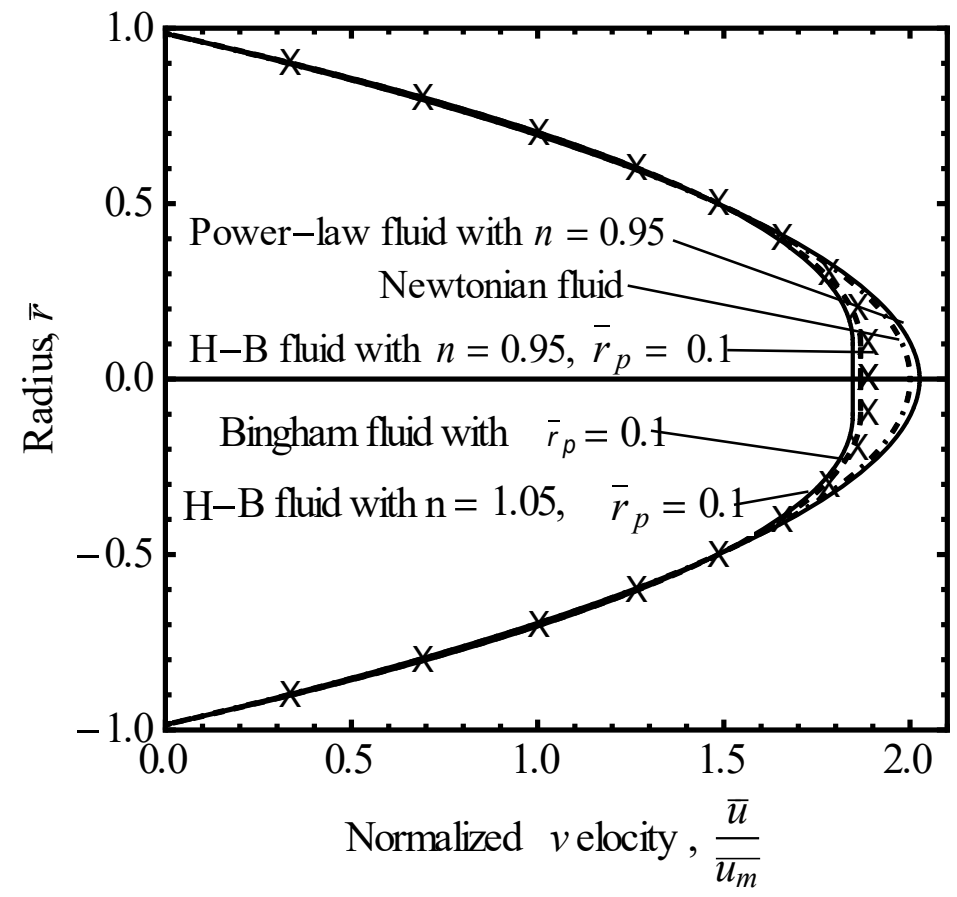

Figure 2. Variation of normalised velocity profiles of different fluids when $\overline{\bar{l}}_{0}=3, \bar{d}=2, \bar{z}=4$ and $\bar{\delta}=$ 0.01 .

\section{Relative Axial Diffusivity}

The variation in the relative axial diffusivity of the solute against plug core radius, $r_{p}$ for different values of chemical reaction rate parameter, stenosis height, stenosis length, and power-law index are shown in Figure 3,

Figure 4, and Figure 5, respectively. This subsection presents the behaviour of the chemical reaction parameter rate and its effects on the relative axial diffusivity. The variation of relative axial diffusivity of the solute against plug core radius, $r_{p}$ for different values of chemical reaction rate parameter and stenosis height, is illustrated in Figure 3. The effect of the relative axial diffusivity on the stenosis height, $\delta$ is significant to determine the size of stenosis. As the stenosis height increases, the stenosed artery becomes narrower and affects the normal blood flow in the artery. It also delineates that an increase in the chemical reaction rate parameter and stenosis height will result in the decrease of relative axial diffusivity of the solute due to the reduction of the blood viscosity. When the chemical reaction rate parameter increases, more solutes react with the fluid, thus the dispersion of solute decreases.

Figure 4 displays the variation of relative axial diffusivity of the solute against plug core radius, $r_{p}$ for different values of chemical reaction rate parameter and stenosis length. As the length of stenosis, $l_{0}$ increases from 4 to 6 , while the other parameters, $\delta=0.1, d=2$, and $z=4$ stay constant, the radius of the stenosed artery, $R(z)$ monotonically increases. The rise in the plug core radius can increase the viscosity of the fluid, therefore, minimises the relative axial diffusivity of the solute. It is postulated that 
the increase in the chemical reaction rate parameter decreases the relative axial diffusivity slowly as the length of stenosis decreases because of the increase in the amount of solute that undergoes the chemical reaction in the bulk flow. The variation of relative axial diffusivity of the solute against plug core radius, $r_{p}$ for different values of power-law index and chemical reaction rate parameter is shown in Figure 5. The analysis shows that when the chemical reaction rate parameter increases, the relative axial diffusivity of solute decreases as the power-law index increases. This is because viscosity is an essential influence of the power-law index on the velocity profile. In other words, it is velocity gradient-dependent. Physically, the velocity gradient decreases with the increase of the power-law index, and the viscosity increases. When the viscosity increases in the blood flow, the solute movement becomes slower, and hence, the relative axial diffusivity decreases.

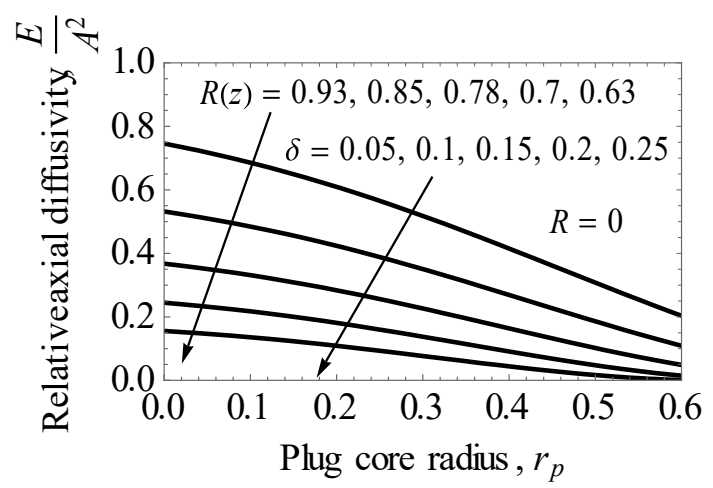

(a)

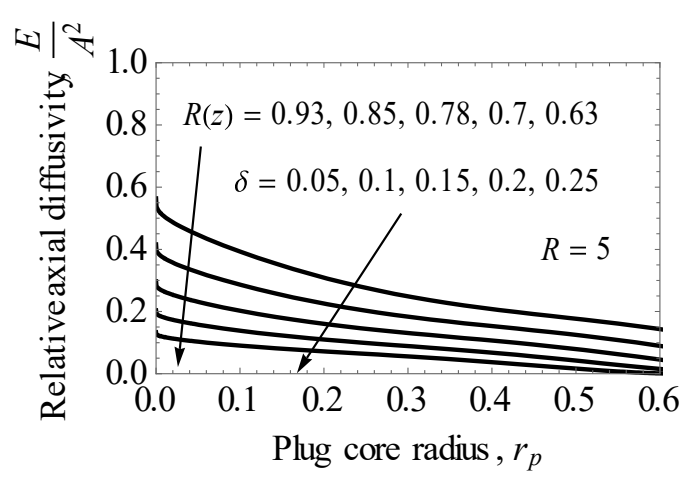

(c)

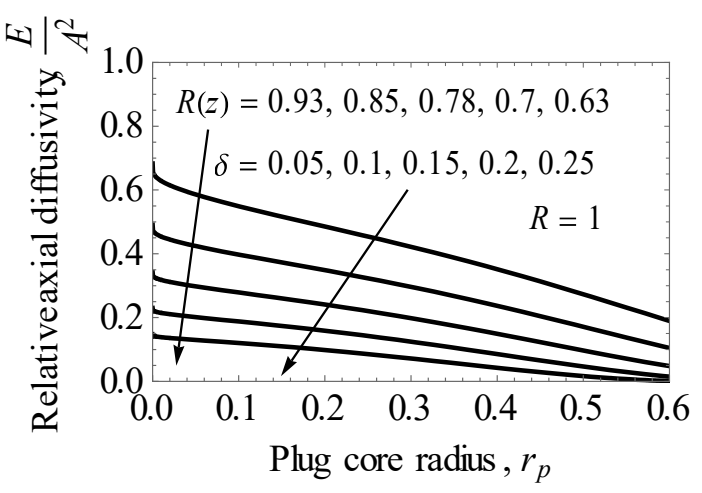

(b)

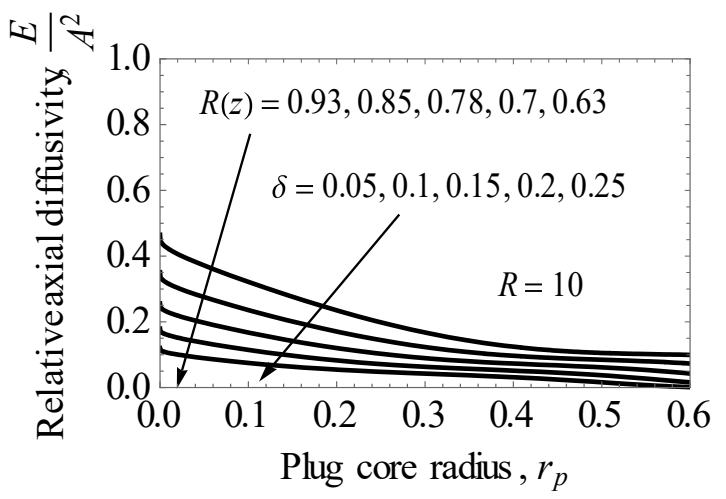

(d)

Figure 3. Variation of the relative axial diffusivity of the solute against plug core radius, $r_{p}$ for different values of stenosis height and chemical reaction rate parameter (a) $R=0$, (b) $R=1$, (c) $R=5$, and (d) $R=10$ when $l_{0}=3, d=2, n=0.95$, and $z=4$.

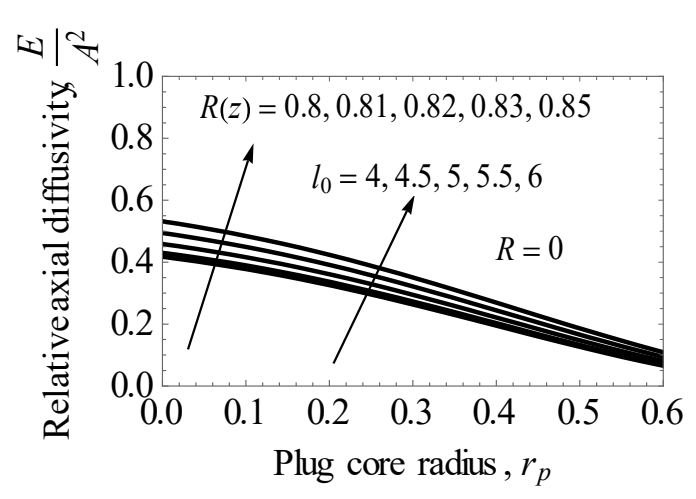

(a)

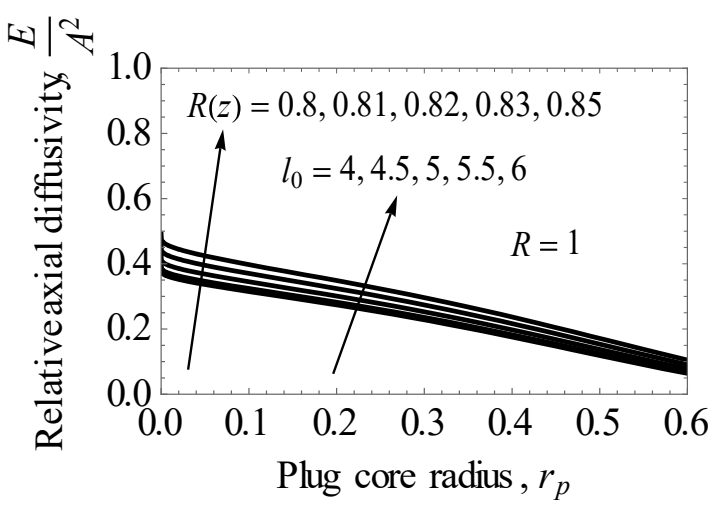

(b) 


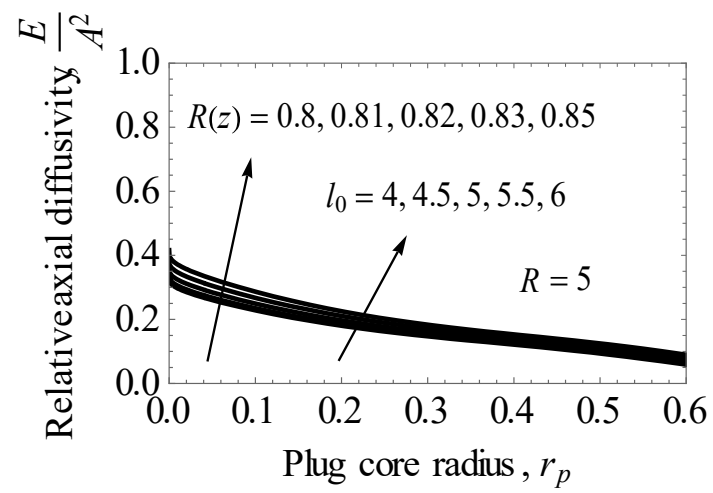

(c)

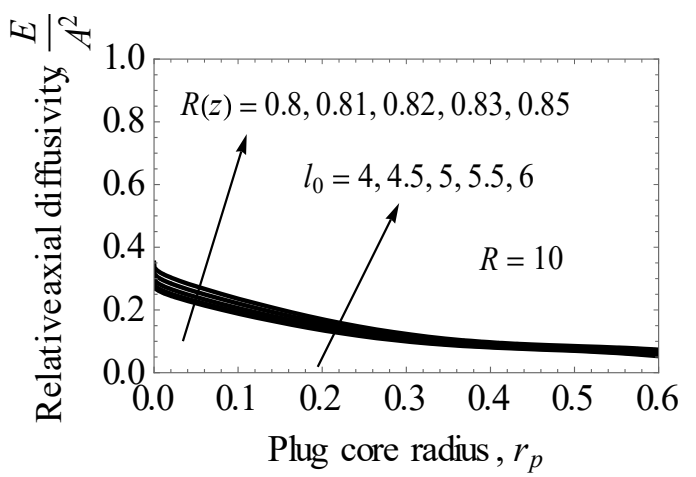

(d)

Figure 4. Variation of the relative axial diffusivity of the solute against plug core radius, $r_{p}$ for different values of stenosis length and chemical reaction rate parameter (a) $R=0$, (b) $R=1$, (c) $R=5$, and (d) $R=10$ when $\delta=0.1, d=2, n=0.95$, and $z=4$.

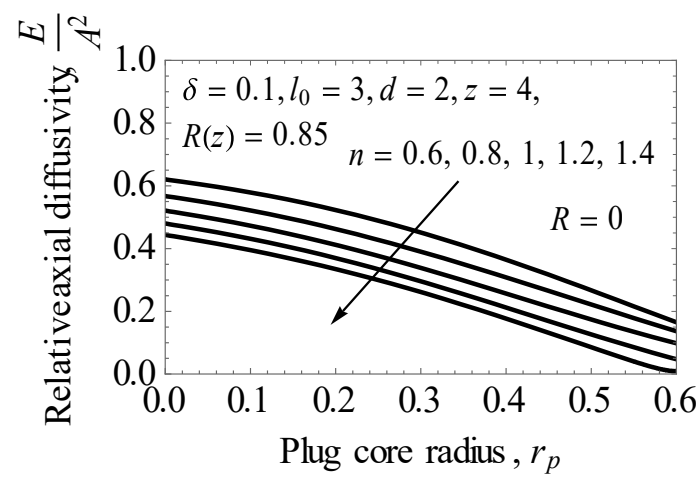

(a)

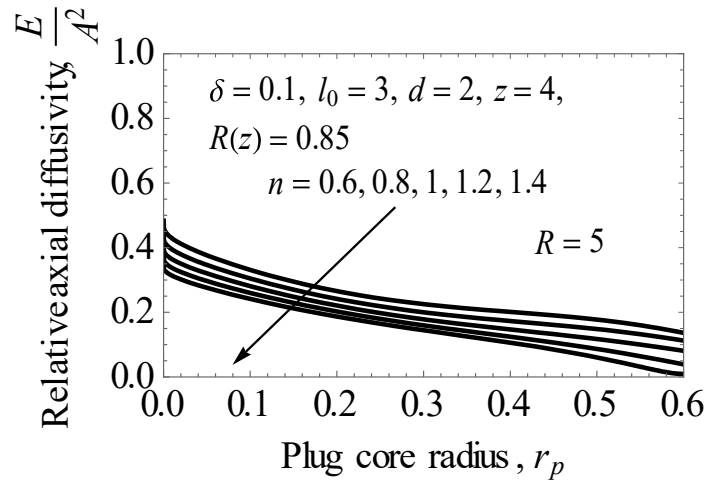

(c)

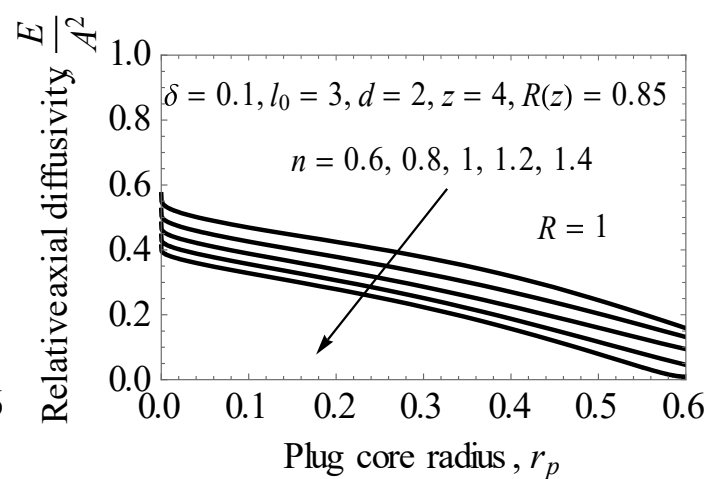

(b)

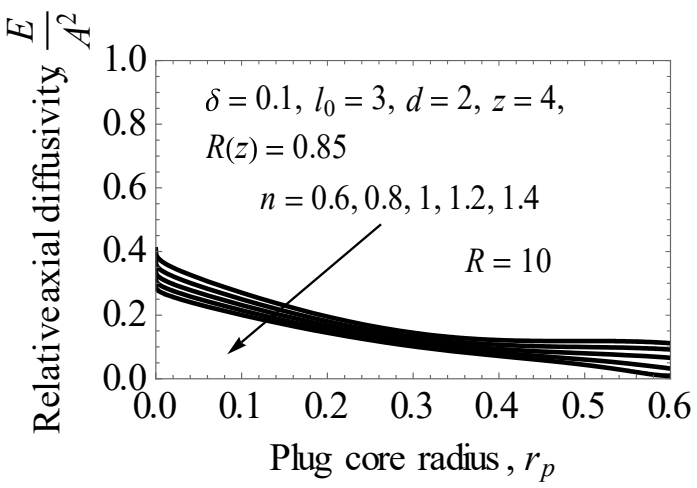

(d)

Figure 5. Variation of the relative axial diffusivity of the solute against plug core radius, $r_{p}$ for different values of the power-law index and chemical reaction rate parameter (a) $R=0$, (b) $R=1$, (c) $R=5$, and (d) $\mathrm{R}=10$ when $\delta=0.1, d=2, l_{0}=3$, and $z=4$.

\section{Effective Axial Diffusivity}

Figure 6 demonstrates the variation of the effective axial diffusivity of a solute against plug core radius, $r_{p}$ for different values of Péclet number and chemical reaction rate parameter when $\delta=0.1, d=2, l_{0}=$ $5, n=0.95$ and $z=4$. It is found that at lower values of Péclet number ( $\mathrm{Pe}=4$ and 8 ), the effective axial diffusivity decreases slowly (almost constant) but at higher values of Péclet number $(\mathrm{Pe}=12,16$, and 20 ), it decreases considerably with an increase in the yield stress. One possible reason is the increase 
of the red blood cells at the centre that reduces the velocity of blood, hence slowing down the effective axial diffusivity of solute. It is also noted that the effective axial diffusivity increases with the increase of the Péclet number. When the Péclet number increases, the convection of solute in the blood flow is dominant compared to diffusion at the early stage of intravenous medication. So, the concentration of medicine is higher when arrived at the targeted area, thus explaining the higher effective axial diffusivity of solute at the targeted area. Jaafar [40] stated that when $P e=0$, the effective axial diffusivity is constant and no changes occur with yield stress because the diffusion takes place in the overall dispersion process as compared to convection.

Next, the variation of the effective axial diffusivity of a solute against plug core radius, $r_{p}$ for different values of power-law index and chemical reaction rate parameter when $\delta=0.1, d=2, l_{0}=3$, and $z=4$ are illustrated in Figure 7. As mentioned by Hussain et al. [41], the power-law index represents the apparent whole blood viscosity. When the power-law index decreases, the viscosity decreases, thus the blood moves slower along with the axial distance and produced an increase in the effective axial diffusivity of solute. Figure 8 depicts the variation of the effective axial diffusivity of a solute against plug core radius, $r_{p}$ for different values of stenosis height and chemical reaction rate parameter when $n=$ $0.95, d=2, l_{0}=3$, and $z=4$. As the stenosis height increases, the stenosed artery becomes narrower due to the accumulation of cholesterols, fats, lipids, and other unwanted substances at the arterial wall, ultimately disrupting the blood flow. It is also observed that the effective axial diffusivity decreases when the chemical reaction and stenosis height increase. The degree of solute binding to blood proteins affects the effectiveness of solute dispersion. An increase in the chemical reaction between the solute and blood in the blood flow will increase the number of molecules that undergo the chemical reaction, at the same time reducing the solute concentration. Hence, a decrease in the solute concentration causes the effective axial diffusivity of solute to decrease as the stenosis height increases. The bound solutes are inactive. Only the free and, unbound solutes can react at the target sites in the tissues.

The estimates of the effective axial diffusivity of solute in $\mathrm{H}-\mathrm{B}$ fluid flow for different values of stenosis length, $l_{0}$ when the chemical reaction rate parameter, $R=5$ is presented in Table 1 . It is observed that the effective axial diffusivity of the solute decreases slowly with an increase in the yield stress. Increasing yield stress causes the accumulation of red blood cells at the centre, hence reducing the blood velocity. As the stenosis length increase, the effective axial diffusivity of the solute decreases marginally.

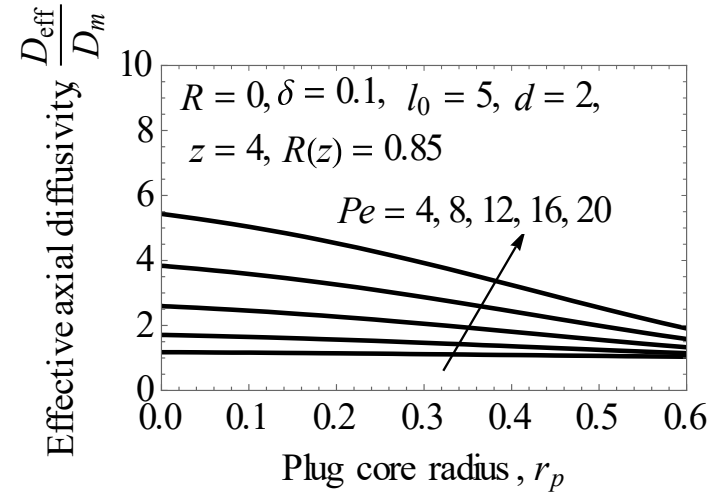

(a)

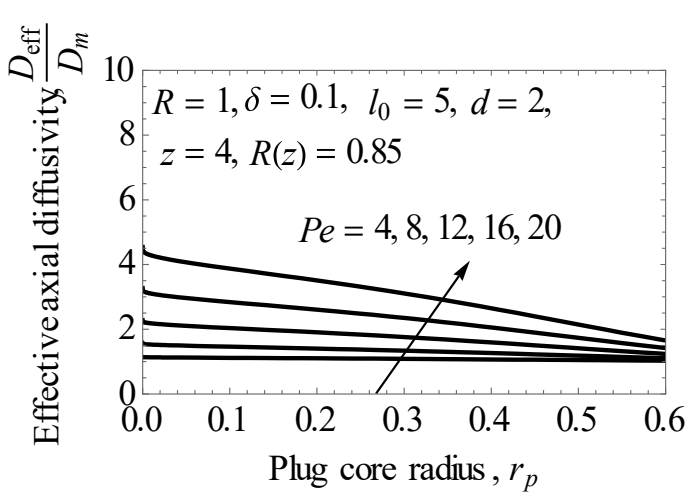

(b) 


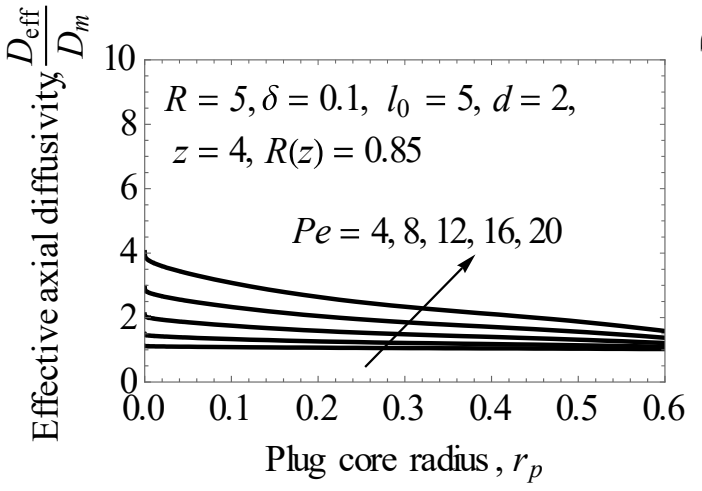

(c)

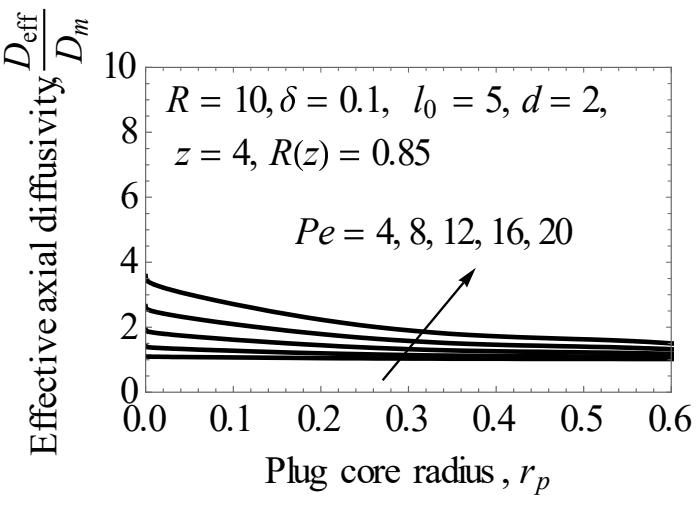

(d)

Figure 6. Variation of the effective axial diffusivity of the solute against plug core radius, $r_{p}$ for different values of the Péclet number and chemical reaction rate parameter (a) $R=0$, (b) $R=1$, (c) $R=5$, and (d) $\mathrm{R}=10$ when $\delta=0.1, d=2, l_{0}=5, n=0.95$, and $z=4$.
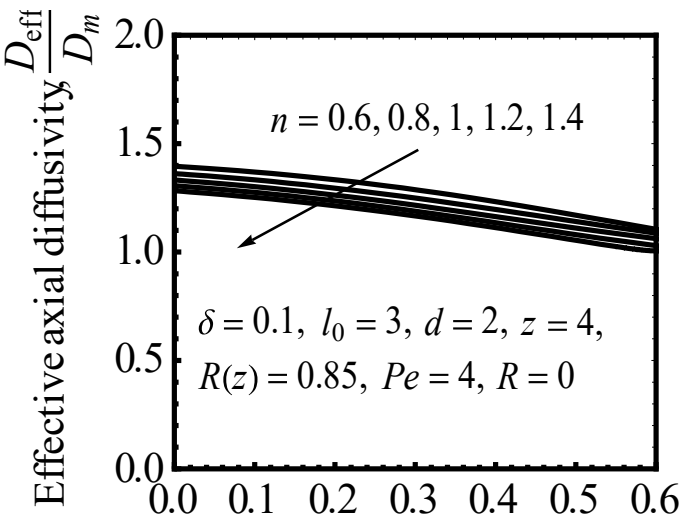

Plug core radius, $r_{p}$

(a)

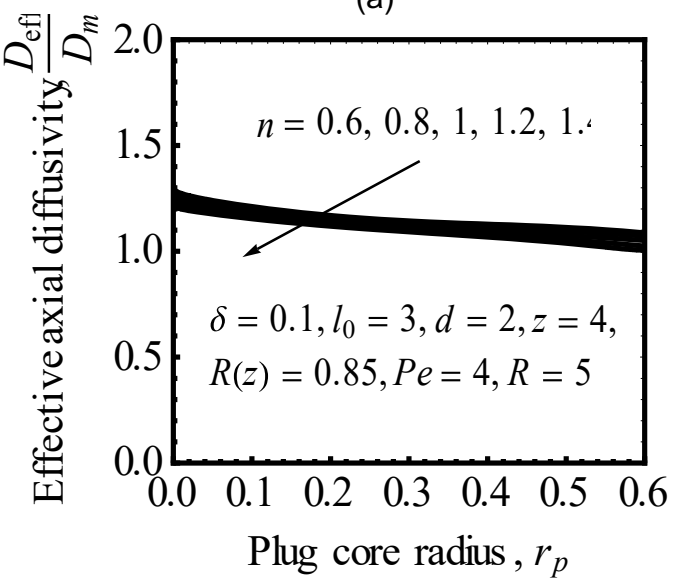

(c)

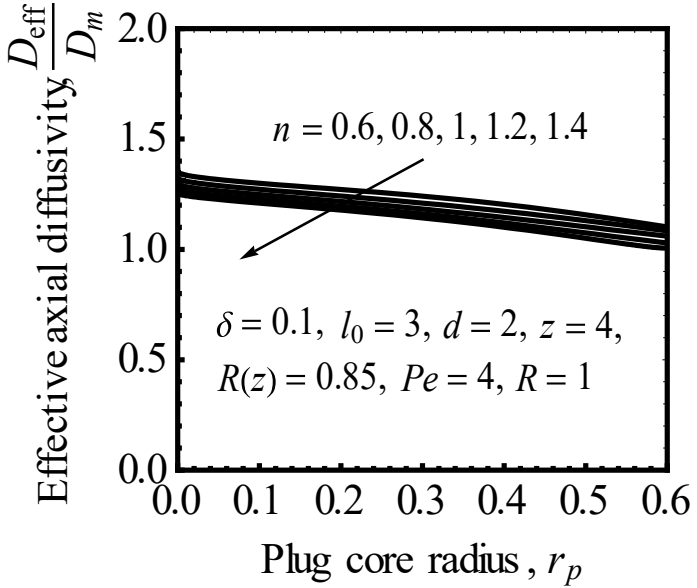

(b)
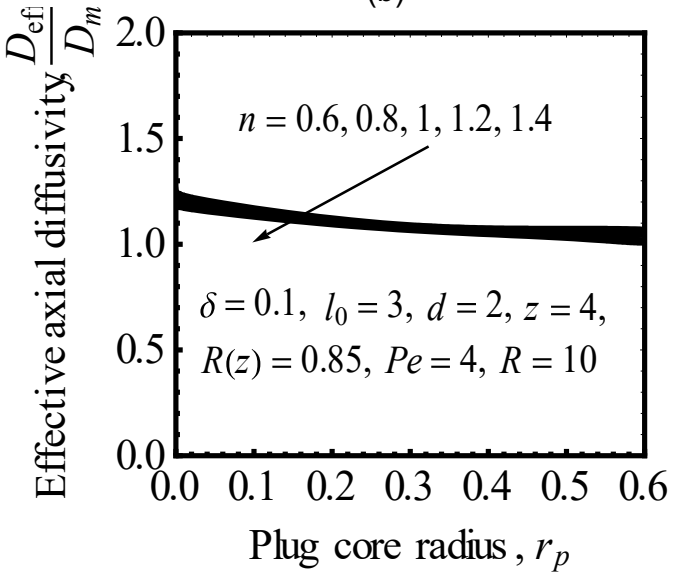

(d)

Figure 7. Variation of the effective axial diffusivity of the solute against plug core radius, $r_{p}$ for different values of the power-law index and chemical reaction rate parameter (a) $R=0$, (b) $R=1,(c) R=5$, and (d) $\mathrm{R}=10$ when $\delta=0.1, d=2, l_{0}=3$, and $z=4$. 


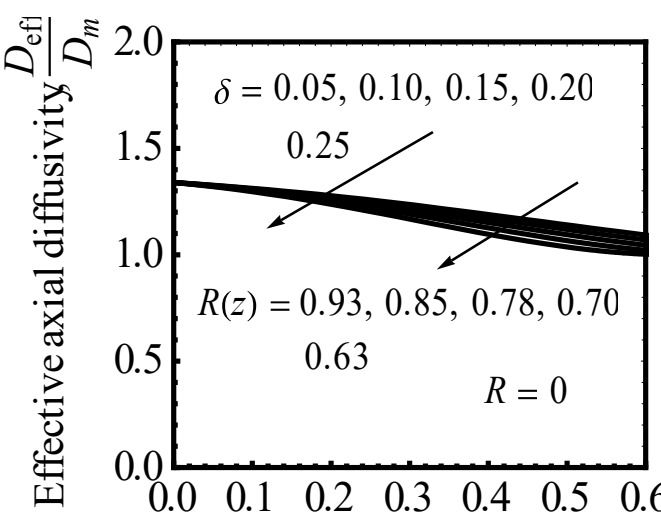

Plug core radius, $r_{p}$

(a)

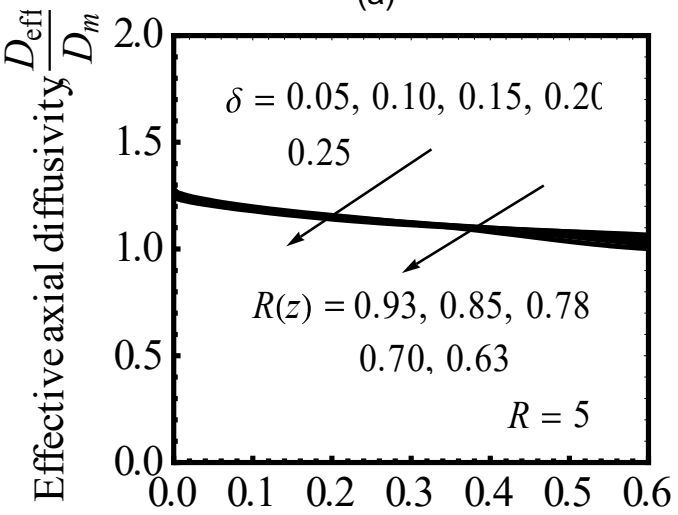

Plug core radius, $r_{p}$

(c)

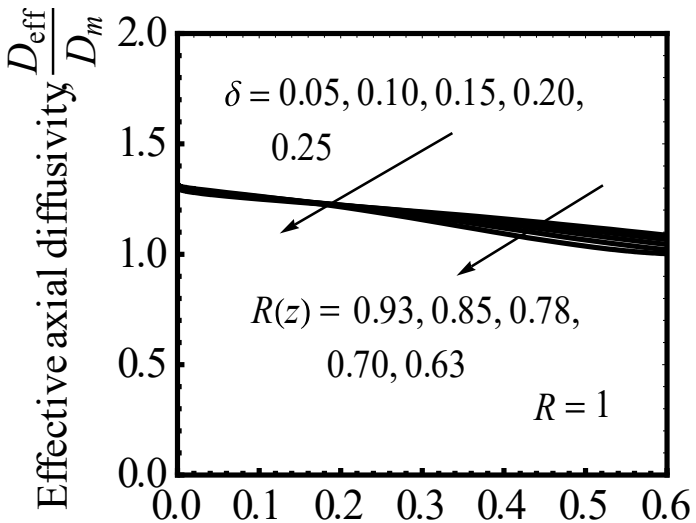

Plug core radius, $r_{p}$

(b)

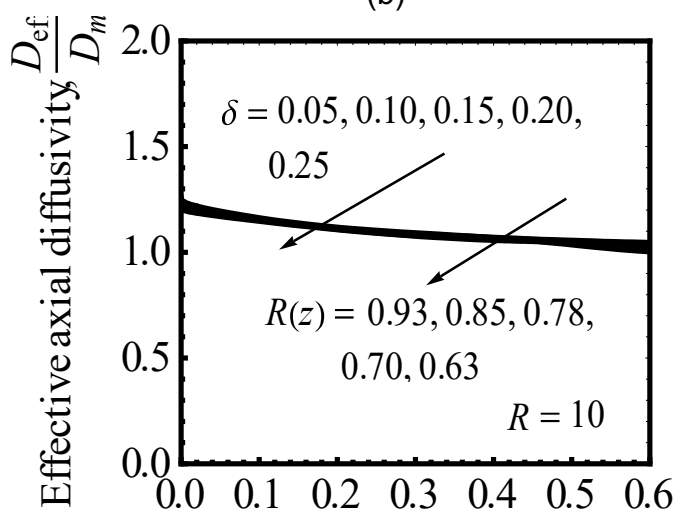

Plug core radius, $r_{p}$

(d)

Figure 8. Variation of the effective axial diffusivity of the solute against plug core radius, $r_{p}$ for different values of the stenosis height and chemical reaction rate parameter (a) $R=0$, (b) $R=1$, (c) $R=5$, and (d) $\mathrm{R}=10$ when $d=2, l_{0}=3, n=0.95$, and $z=4$.

Table 1. Estimates of the effective axial diffusivity of solute in $\mathrm{H}-\mathrm{B}$ fluid flow for different values of stenosis length, $l_{0}$ when the chemical reaction rate parameter, $R=5$

\begin{tabular}{cccccc}
\hline \multirow{2}{*}{ Plug core radius, $r_{p}$} & \multicolumn{5}{c}{$1+\frac{P e^{2}}{48}\left(\frac{E\left(r_{p}\right)}{A^{2}\left(r_{p}\right)}\right)\left(\frac{1}{R(z)^{4}}\right)$} \\
\cline { 2 - 6 } & $l_{0}=4.0$ & $l_{0}=4.5$ & $l_{0}=5.0$ & $l_{0}=5.5$ & $l_{0}=6.0$ \\
\hline $10^{-8}$ & 1.272 & 1.271 & 1.270 & 1.268 & 1.266 \\
\hline 0.1 & 1.186 & 1.187 & 1.186 & 1.185 & 1.184 \\
\hline 0.2 & 1.147 & 1.146 & 1.144 & 1.143 & 1.142 \\
\hline 0.3 & 1.120 & 1.119 & 1.118 & 1.116 & 1.115 \\
\hline 0.4 & 1.099 & 1.099 & 1.099 & 1.098 & 1.098 \\
\hline 0.5 & 1.076 & 1.077 & 1.078 & 1.079 & 1.080 \\
\hline 0.6 & 1.048 & 1.050 & 1.052 & 1.054 & 1.057 \\
\hline
\end{tabular}

\section{Real clinical applications}

Figure 9 displays the variation of normalised velocity profiles for different types of arteries when $\bar{\delta}=$ 
$0.01,0.02,003,0.04$ and 0.05 . The physiological data of some blood vessels with their diameters in Das and Saha [42] were used in this study to estimate the normalised velocity profiles of the solute in blood flow. The blood velocity can be studied by analysing the normalised velocity with equation (36). As mentioned by Sharp [21], the fully developed velocity profile is not parabolic but it exhibits a core region of constant velocity and an outer region with a velocity gradient as shown in

Figure 9. As the stenosis height increases, the normalised velocity profiles decrease marginally with a decreasing diameter of the blood vessels. The blood moves slower along the axial distance as the velocity decreases. In the aorta, the flow initially increasing until $\bar{r}=0.2$, after that the flow experiences a core region before decreasing at $\bar{r}=-0.2$ until it reaches the minimum velocity. In the femoral artery, the flow increases until $\bar{r}=0.13$, then the flow experiences a core region and starts to decrease at $\bar{r}=$ -0.13 until it reaches the minimum velocities.

Meanwhile, in carotid artery, at the beginning, the flow also increases until $\bar{r}=0.1$. Then, it experiences a core region and starts to decrease at $\bar{r}=-0.1$ until it reaches the minimum velocities. The maximum velocities of the blood vessels are $1.83-1.87 \mathrm{~cm} \mathrm{~s}^{-1}, 1.62-1.72 \mathrm{~cm} \mathrm{~s}^{-1}$, and $1.42-1.62 \mathrm{~cm} \mathrm{~s}^{-1}$ respectively, with an increase in the stenosis height. At the maximum radius, the normalised velocity, $\bar{u} / \bar{u}_{m}=0$ due to the effects of vessel walls on the blood fluid and the adhesion of blood fluid particles to the boundary wall of the vessel under no-slip condition. Bessonov et al. [31] stated that the erythrocyte concentration is greater near the axis, while platelets are more commonly localised near the wall. The velocity profiles are parabolic in simulations that involve only plasma particles. However, in simulations with erythrocytes, the velocity profiles are flat in the middle of the vessel because of the high erythrocyte concentration.

The variation of the relative axial diffusivity and effective axial diffusivity of solute in blood vessels when $\bar{\delta}=0.08, \bar{r}_{p}=0.2, \bar{d}=2, \bar{l}_{0}=3, n=0.95$, and $\bar{z}=4$ is tabulated in Table 2 . Aorta, the largest artery that carries oxygenated blood away from the heart to all parts of the body, has the largest diameter with a radius of $1 \mathrm{~cm}$. Meanwhile, the femoral and carotid arteries have a radius of $0.5 \mathrm{~cm}$ and $0.4 \mathrm{~cm}$, respectively. As seen in Table 2, as the radius of the artery decreases, the axial diffusivity of the solute also decreases. Theoretically, it implies that the chemical reaction that occurs between the medicine and blood proteins tends to decrease the relative diffusivity of the solute. These results reveal that since the chemical reaction rate parameter is considered, a number of medicine molecules that undergo the protein binding decreases the relative diffusivity of solute. The degree of binding of the solute to blood proteins also affects the effectiveness of solute dispersion. This is because an increased chemical reaction between the solute and blood in the blood flow tends to increase the number of molecules that undergo the chemical reaction, thus reducing the amount of solute concentration. Hence, the decrease in the solute concentration causes the effective axial diffusivity of the solute to decrease. The highest level of effective diffusivity of solute in the blood flow shows that the medicine in the blood flow achieves the highest efficiency.

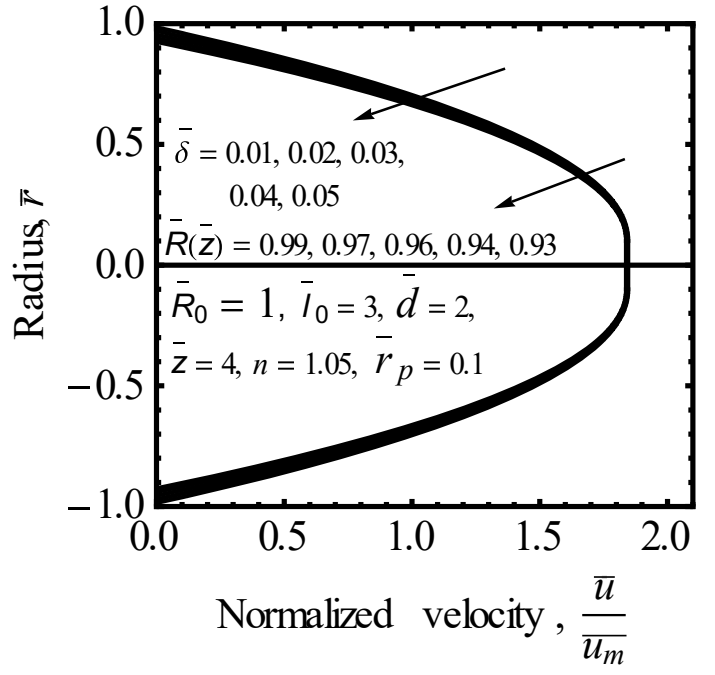

(a)

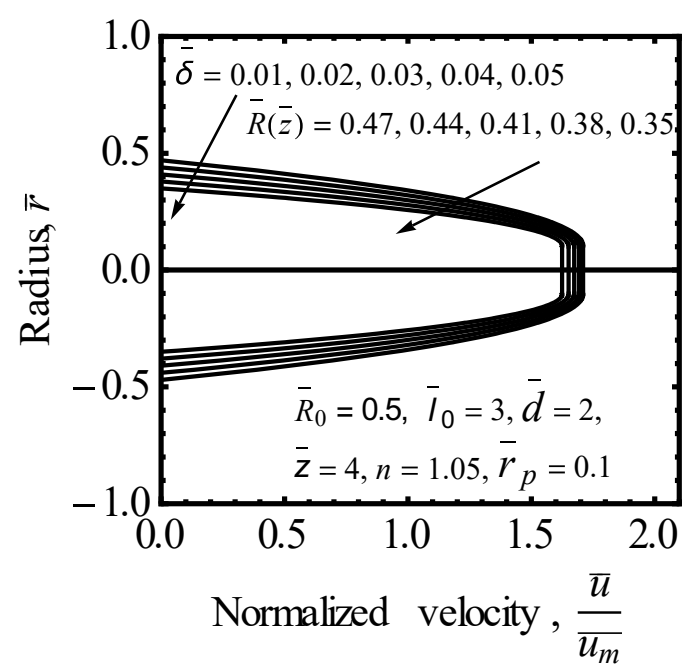

(b) 


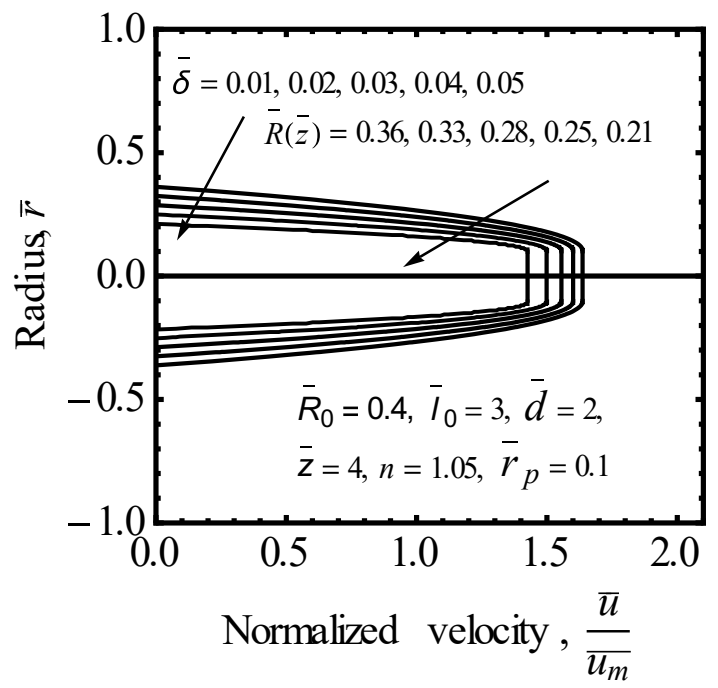

(c)

Figure 9. Variation of normalised velocity profiles against radial coordinate when $\bar{\delta}=0.01,0.02$, $0.03,0.04$ and 0.05 for (a) aorta, (b) femoral artery, and (c) carotid artery.

Table 2. Variation of the relative axial diffusivity and effective axial diffusivity of solute in blood vessels when $\bar{\delta}=0.08, \bar{r}_{p}=0.2, \bar{d}=2, \bar{l}_{0}=3, n=0.95, \bar{R}=5$, and $\bar{z}=4$.

\begin{tabular}{ccccc}
\hline Blood vessels & Radius $(\mathrm{cm})$ & $\frac{E\left(\bar{r}_{p}\right)}{A^{2}\left(\bar{r}_{p}\right)}$ & $1+\frac{P e^{2}}{48}\left(\frac{E\left(\bar{r}_{p}\right)}{A^{2}\left(\bar{r}_{p}\right)}\right)\left(\frac{1}{\bar{R}(\bar{z})^{4}}\right)$ & $\bar{R}(\bar{z})(\mathrm{cm})$ \\
\hline Aorta & 1.0 & 0.454 & 1.151 & 0.88 \\
Femoral artery & 0.5 & 0.008 & 1.003 & 0.38 \\
Carotid artery & 0.4 & 0.001 & 1.000 & 0.28 \\
\hline
\end{tabular}

\section{Conclusion}

In summary, the problem of steady dispersion of a solute in blood flow with the inclusion of reactive species in a stenosed artery can be solved by varying the physical parameters. The yield stress plays an essential role in the determination of blood viscosity and velocity profiles in the blood flow. The normalised velocity of blood, relative axial diffusivity, and effective axial diffusivity of solute decrease with an increase in the power-law index and yield stress of the blood flow. Thus, the power-law index and yield stress affect the flow behaviour of blood. When the power-law index and yield stress increase, the concentration of red blood cells at the centre also increases. Thus, the velocity of blood, relative axial diffusivity, and effective axial diffusivity of solute decrease. The stenosis height, stenosis length, and power-law index increase with an increased chemical reaction rate parameter while the relative axial diffusivity of solute shows a reversed behaviour. The increase of the chemical reaction rate parameter reduces the concentration of solute. The decrease of the solute concentration can be due to the presence of chemical reactions that can lead to the consumption or destruction of chemical species. The effective axial diffusivity increases considerably with an increase in the Péclet number because the solute movement to the targeted area is higher, thus, increasing the diffusivity of solute. The increment in stenosis height, stenosis length, and power-law index also reduces the effective axial diffusivity of solute. From the results obtained in this mathematical analysis, there is a significant difference between the flow quantities computed in the presence of chemical reaction and stenosis when compared with an absence of chemical reaction and stenosis. Hence, our mathematical analysis contributes significant improvement to the field of mathematical modelling of the steady dispersion of a solute in blood flow through narrow arteries in the presence of chemical reactions and stenosis. 


\section{Conflicts of interest}

The authors declare that there is no conflict of interest regarding the publication of this paper.

\section{Funding statement}

The research and publication of this article were funded by the Ministry of Higher Education (MOHE) and Research Management Centre, Universiti Teknologi Malaysia [Q. J130000.2654.17J12] (GUP Tier 2 Grant), [Q. J130000.2554.21H48] (UTMFR Grant) and [R. J130000.7854.5F255] (FRGS Grant).

\section{Acknowledgments}

The author would like to acknowledge the support and hospitality of the Sydney Mathematical Research Institute (SMRI).

\section{References}

[1]. De Carli, J. P., Carta, G., \& Byers, C. H. (1990). Displacement separations by continuous annular chromatography. AlChE journal, 36(8), 1220-1228.

[2]. Vikhansky, A., \& Wang, W. (2011). Taylor dispersion in finite-length capillaries. Chemical Engineering Science, 66(4), 642-649.

[3]. Agrawal, S., \& Jayaraman, G. (1994). Numerical simulation of dispersion in the flow of power law fluids in curved tubes. Applied Mathematical Modelling, 18(9), 504-512.

[4]. Mercer, G., \& Roberts, A. (1990). A centre manifold description of contaminant dispersion in channels with varying flow properties. SIAM Journal on Applied Mathematics, 50(6), 1547-1565.

[5]. Taylor, G. I. (1953). Dispersion of soluble matter in solvent flowing slowly through a tube. Proceedings of the Royal Society of London. Series A. Mathematical Physical Sciences, 219(1137), 186-203.

[6]. Aris, R. (1956). On the dispersion of a solute in a fluid flowing through a tube. Proceedings of the Royal Society of London. Series A. Mathematical Physical Sciences, 235(1200), 67-77.

[7]. Ali, N., Zaman, A., Sajid, M., Nieto, J., and Torres, A. (2015). Unsteady non-Newtonian blood flow through a tapered overlapping stenosed catheterized vessel. Mathematical Biosciences, 269, 94-103.

[8]. Sriyab, S. (2014). Mathematical analysis of non-Newtonian blood flow in stenosis narrow arteries. Computational mathematical methods in medicine, 2014.

[9]. Shah, S. R. (2011). Study of modified Casson's fluid model in modeled normal and stenotic capillary-tissue diffusion phenomena. International Journal of Computational Engineering and Management, 11, 51-57.

[10]. Mishra, S., and Siddiqui, S. (2015). A mathematical model for flow and diffusion through stenotic capillary-tissue exchange system. Journal of Science and Technology, 24(4), 321-337.

[11]. Bhatnagar, A., Kumar, V., \& Shrivastav, R. (2018). Blood Flow in a Radially Non Symmetric Stenosed Artery Under Slip Effect Through Porous Medium. National Academy Science Letters, 41(6), 349-353.

[12]. Majee, S., \& Shit, G. (2020). Modeling and simulation of blood flow with magnetic nanoparticles as carrier for targeted drug delivery in the stenosed artery. European Journal of Mechanics-B/Fluids, 83, 42-57.

[13]. Haghighi, A. R., \& Aliashrafi, N. (2018). A mathematical modeling of pulsatile blood flow through a stenosed artery under effect of a magnetic field. Journal of Mathematical Modeling, 6(2), 149-164.

[14]. Shahzadi, I., \& Bilal, S. (2020). A significant role of permeability on blood flow for hybrid nanofluid through bifurcated stenosed artery: Drug delivery application. Journal Computer Methods Programs in Biomedicine, 187, 105248.

[15]. Freidoonimehr, N., Chin, R., Zander, A., \& Arjomandi, M. (2020). An experimental model for pressure drop evaluation in a stenosed coronary artery. Journal Physics of Fluids, 32(2), 021901.

[16]. Kori, J. (2020). Effect of first order chemical reactions on the dispersion coefficient associated with laminar flow through fibrosis affected lung. Journal of biomechanics, 99, 109494

[17]. Tal, A. (2006). Simplified models for gas exchange in the human lungs. Journal of theoretical biology, 238(2), 474-495.

[18]. Bird, R. B. (1979). Transport phenomena. J Appl. Mech. Rev., 55(1).

[19]. Ratchagar, N. P., and VijayaKumar, R. (2019). Dispersion of solute with chemical reaction in blood flow. Bulletin of Pure and Applied Sciences-Mathematics and Statistics, 38(1), 385-395.

[20]. Mwapinga, A., Mureithi, E., Makungu, J., and Masanja, V. (2020). MHD Arterial Blood Flow and Mass Transfer under the Presence of Stenosis, Body Acceleration and Chemical Reaction: A Case of Magnetic Therapy. Journal of Mathematics and Informatics, 18, 85-103.

[21]. Sharp, M. K. (1993). Shear-augmented dispersion in non-Newtonian fluids. Annals of Biomedical Engineering, 21(4), 407-415.

[22]. Tu, C., and Deville, M. (1996). Pulsatile flow of non-Newtonian fluids through arterial stenoses. Journal of biomechanics, 29(7), 899-908.

[23]. Mazumdar, J. (1992). Biofluid Mechanics World Scientific. In: Singapore London.

[24]. Jaafar, N. A., Yatim, Y. M., and Sankar, D. (2017). Effect of chemical reaction in solute dispersion in Herschel- 
Bulkley fluid flow with applications to blood flow. Advances and Applications in Fluid Mechanics, 20(2), 279310.

[25]. Al Mukahal, F., Duffy, B., \& Wilson, S. (2017). Advection and Taylor-Aris dispersion in rivulet flow. Proceedings of the Royal Society A: Mathematical, Physical Engineering Sciences, 473(2207), 20170524.

[26]. Salerno, L., Cardillo, G., \& Camporeale, C. (2020). Aris-Taylor dispersion in the subarachnoid space. Physical Review Fluids, 5(4), 043102

[27]. Westerbeek, E. Y., Bomer, J. G., Olthuis, W., Eijkel, J. C., \& De Malsche, W. (2020). Reduction of Taylor-Aris dispersion by lateral mixing for chromatographic applications. Lab on a Chip, 20(21), 3938-3947.

[28]. Swarup, S. (2000). Fluid Dynamics, Krishna Prakashan Media (P) Ltd. Merut, 622, 633.

[29]. White, F. M. (2011). Fluid mechanics. 7th edition. New York, McGraw Hill.

[30]. Singh, A., Shrivastav, R., and Bhatnagar, A. (2015). A numerical analysis for the effect of slip velocity and stenosis shape on Non-Newtonian flow of blood. International Journal of Engineering, 28(3), 440-446.

[31]. Bessonov, N., Sequeira, A., Simakov, S., Vassilevskii, Y., and Volpert, V. (2016). Methods of blood flow modelling. J Mathematical Modelling of Natural Phenomena, 11(1), 1-25.

[32]. Chitra, M., \& Karthikeyan, D. (2018). Mathematical modeling of power law and Herschel-Bulkley non-Newtonian fluid of blood flow through a stenosed artery with permeable wall: effects of slip velocity. Paper presented at the Journal of Physics: Conference Series.

[33]. Sankar, D., \& Lee, U. (2008). Two-fluid Herschel-Bulkley model for blood flow in catheterized arteries. Journal of Mechanical science technology, 22(5), 1008.

[34]. Misra, J., \& Shit, G. (2006). Blood flow through arteries in a pathological state: A theoretical study. International Journal of Engineering Science, 44(10), 662-671.

[35]. Shukla, J., Parihar, R., \& Rao, B. (1979). Dispersion in non-Newtonian fluids: Effects of chemical reaction. Rheologica Acta, 18(6), 740-748.

[36]. Singh, S., Chadda, G., \& Sinha, A. (1989). A study of sectionally related dispersion and chemical reaction effects. Defence Science Journal, 39(3), 305.

[37]. Sankar, D., \& Lee, U. (2009). Mathematical modeling of pulsatile flow of non-Newtonian fluid in stenosed arteries. Communications in Nonlinear Science Numerical Simulation, 14(7), 2971-2981.

[38]. Gentile, F., Ferrari, M., \& Decuzzi, P. (2008). The transport of nanoparticles in blood vessels: the effect of vessel permeability and blood rheology. $J$ Annals of biomedical engineering, 36(2), 254-261.

[39]. Sankar, D., Jaafar, N. A., and Yatim, Y. M., (2012). Nonlinear analysis for shear augmented dispersion of solutes in blood flow through narrow arteries. Journal of Applied Mathematics, 20(12), 201-222.

[40]. Nurul Aini binti Jaafar (2017) Mathematical Analysis of Herschel-Bulkley Fluid Model for Solute Dispersion in Blood Flow through Narrow Conduits. PhD Thesis, Universiti Sains Malaysia, Penang.

[41]. Hussain, M. A., Kar, S., \& Puniyani, R. R. (1999). Relationship between power law coefficients and major blood constituents affecting the whole blood viscosity. J Journal of Biosciences, 24(3), 329-337.

[42]. Das, P., Sarifuddin, M., \& Kumar, P. (2020). Solute dispersion in Casson fluid flow through a stenosed artery with absorptive wall. Journal of Zeitschrift Angewandte Mathematik und Physik, 71(3), 100. 